\title{
Mapping Anatomy to Behavior in Thy1:18 ChR2-YFP Transgenic Mice Using Optogenetics
}

\author{
Lief E. Fenno, ${ }^{1}$ Lisa A. Gunaydin, ${ }^{2}$ and Karl Deisseroth ${ }^{3,4}$ \\ ${ }^{1}$ Stanford Neuroscience and Medical Scientist Training Programs, Stanford University, Stanford, California \\ 94305; ${ }^{2}$ Gladstone Institute of Neurological Disease, University of California San Francisco, San Francisco, \\ California 94158; ${ }^{3}$ Howard Hughes Medical Institute and Stanford Bioengineering and Psychiatry, Stanford \\ University, Stanford, California 94305
}

Linking the activity of defined neural populations with behavior is a key goal of neuroscience. In the context of controlling behavior, electrical stimulation affords researchers precision in the temporal domain with gross regional specificity, whereas pharmacology allows for more specific manipulation of cell types, but in the absence of temporal precision. The use of microbial opsins-light activated, genetically encoded ion channels and pumps-to control mammalian neurons now allows researchers to "sensitize" genetically and/or topologically defined populations of neurons to light to induce either depolarization or hyperpolarization in both a cell-type-specific and temporally precise manner not achievable with previous techniques. Here, we describe the use of transgenic mice expressing the bluelight gated cation channel Channelrhodopsin-2 (ChR2) under control of the Thyl promoter for the purpose of linking neuronal activity to behavior through restricted delivery of light to an anatomic region of interest. The surgical procedure for implanting a fiber-optic light delivery guide into the mouse brain, the process of optically stimulating the brain in a behaving animal, and post hoc evaluation are given, along with necessary reagents and discussion of common technical problems and their solutions.

It is essential that you consult the appropriate Material Safety Data Sheets and your institution's Environmental Health and Safety Office for proper handling of equipment and hazardous material used in this protocol.

RECIPES: Please see the end of this protocol for recipes indicated by $<R>$. Additional recipes can be found online at http://cshprotocols.cshlp.org/site/recipes.

Reagents

Buprenorphine (Buprenex; $0.05 \mathrm{mg} / \mathrm{kg}$ )

Buprenex is an opiate analgesic. It is a controlled substance.

Carprofen (Rimadyl [Pfizer]; $5 \mathrm{mg} / \mathrm{kg}$ )

Carprofen is a nonsteroidal anti-inflammatory drug (NSAID) that is not a controlled substance. It is also available for continued use postoperatively in food pellet form, either in isolation or combined with other drugs (e.g., Carprofen plus the antibiotic enrofloxacin [Baytril] from Bio-Serv).

Dental cements (MetaBond [Parkell S380] and Ortho-Jet [Lang B1323])

The Ortho-Jet resin is optional; see Step 42.

\footnotetext{
${ }^{4}$ Correspondence: deissero@stanford.edu

(C) 2015 Cold Spring Harbor Laboratory Press

Cite this protocol as Cold Spring Harb Protoc; doi:10.1101/pdb.prot075598
} 
L.E. Fenno et al.

Eye lubricant (Altalube [Altaire Pharmaceuticals])

Rodent blinking is suppressed during anesthesia and the eyes must be kept moist at the risk of inducing blindness in the animal. We coat the eyes with a petroleum jelly lubricant during and after surgery.

Isoflurane anesthesia (Butler Schein) and compressed oxygen (Praxair UN1072) Speak with your veterinarian about acquiring both. Isoflurane has several advantages: It quickly brings animals to an operable state, results in a fast recovery period, and is easily titrated during surgery to achieve an acceptable level of anesthesia. Alternatively, ketamine/xylazine anesthetics may be used.

Mice (Thy1[line 18]:ChR2-eYFP)

A good choice are C57/BL6 Thy1 (line 18):ChR2-eYFP mice (JAX Stock No. 007612; Arenkiel et al. 2007) that have enriched expression of ChR2 in cortical layer $V$ and in a number of subcortical structures. Other transgenic mouse lines that directly express opsins are available (Arenkiel et al. 2007; Madisen et al. 2012). Additionally, optogenetic tools may be delivered via viral vectors using promoter-based targeting or an intersectional targeting approach in conjunction with recombinase driver rats and mice (e.g., Cre [Arenkiel et al. 2007; Madisen et al. 2010; Fenno et al. 2011; Witten et al. 2011]). Constructs and ready-made virus are available from the Optogenetics Resource Center (www.optogenetics.org). Opsins may also be delivered to specific cortical layers using in utero electroporation (Adesnik and Scanziani 2010; Gradinaru et al. 2007).

Saline (sterile 0.9\%; AirLife AL4109 [CareFusion])

Equipment

Dental drill and bits (e.g., from Osada or SS White)

Diamond knife (e.g., ThorLabs S90R)

Fiber implants with ferrule (e.g., from ThorLabs or Doric Lenses)

There are multiple sizes and fiber options. MRI-compatible ceramic implants are available.

Fiber-optic patch cable and zirconia sleeve, to connect laser to animal implant (e.g., from Doric Lenses or ThorLabs)

Function generator, to set laser pulse parameters (e.g., Agilent 33220A)

Heating pad for intraoperative temperature regulation (e.g., Kopf TCAT-2LV temperature controller with heating pad)

Isoflurane vaporizer with regulator, induction chamber, tubing, and charcoal filters (e.g., Table Top Laboratory Animal Anesthesia System [VetEquip 901806])

The effects of isoflurane during human pregnancy are not established; speak with your Health and Safety Office before using if pregnant.

Laser (e.g., DPSS [Laserglow] or PhoxX laser [Omicron-Laserage]; see Yizhar et al. 2011)

The ChR2 stimulation peak is blue at $473 \mathrm{~nm}$.

Laser power meter (e.g., from ThorLabs)

Microscope, binocular, with attached or separate illumination (e.g., Leica M60)

Recovery chamber (empty mouse cage with a lamp suspended above)

Stereotactic surgical frame with accessory arm (e.g., Kopf 940)

Surgical tools

Absorption spears (e.g., Fine Science Tools 18105-01 or Harvard Apparatus 598422)

Cotton swabs, sterile (e.g., Puritan HPC25-803)

Forceps (\#3, 5, and 5.5; e.g., Harvard Apparatus 522003 or Fine Science Tools 11251-10)

Bulldog-type hemostatic clamps (e.g., Fine Science Tools 18050-28)

Needle ( 26-gauge)

Needle driver (e.g., Fine Science Tools 12002-12)

Scalpel handle and blades (\#10 and \#11 are popular; e.g., Harvard Apparatus 523522 or Fine Science Tools 10004-13)

Scissors, fine (e.g., Harvard Apparatus 522771 or Fine Science Tools 14090-11)

Scissors for hair removal (veterinary surgical scissors; e.g., Harvard Apparatus 511865 or Fine

Science Tools 14003-16) 
Skin glue (e.g., Vetbond Tissue Adhesive [3M 1469SB])

Standard sterile field

Suture (e.g., Fisher 50-900-04198)

Surgical tool kits (e.g., Harvard Apparatus Mouse Surgical Kit 728943) are available. Reduced-price studentquality surgical equipment is often sufficient for standard surgical procedures.

\section{METHOD}

Experimental animals must be respected. The lives of animals are precious and their well-being is paramount when designing experiments and conducting procedures. Always work under an approved experimental protocol and consult a veterinarian when designing new protocols.

Sterility should be attempted during surgical procedures. While the immune systems of rodents are quite robust, where possible and reasonable, sterile technique should be followed to decrease the potential for infection. Bead sterilizers, autoclaves, and chemical sterilization methods are affordable and generally available.

\section{Identifying the Target Region}

1. Based on the behavior of interest and your hypothesis, use a stereotactic atlas (e.g., Allen Brain Atlas [www.brain-map.org]) to identify the anterior/posterior (A/P), medial/lateral (M/L), and dorsal/ventral (D/V) stereotactic coordinates of a location immediately $(250 \mu \mathrm{m})$ dorsal to the structure to be stimulated.

2. Ensure that Thyl (and thus opsin) is expressed in your anatomic area of interest by referring to published literature, and that lesioning the area within the fiber tract is not likely to compromise the life of the animal.

Choosing coordinates directly on or within $0.2 \mathrm{~mm}$ of the midline will rupture vital vasculature running in an anterior-posterior direction on the surface of the brain and requires alternative targeting strategies beyond the scope of this protocol (see Greenshaw 1997).

Preparing the Surgical Area

3. Clear sufficient space for surgical tools, stereotactic frame, microscope, drill, vaporizer, induction box, and oxygen tank.

4. Prepare surgical equipment: Spread sterile field next to stereotax and assemble sterile surgical equipment, drugs, suture, skin glue, eye lubricant, saline, adsorbent, and small gauge needle.

5. Prepare stereotax: Turn on heating pad and place in animal surgical area, insert ear bars, connect vaporizer outflow tubing to one side of the nosecone and charcoal filter tubing to the other, and attach cannula arm. Turn on drill, attach new drill bit, and position foot pedal.

6. Prepare vaporizer: Check oxygen tank level, open oxygen tank valve, fill isoflurane reservoir, check charcoal canister capacity, and set valves to send vaporizer outflow to induction box.

7. Prepare microscope: Ensure that the microscope will focus on stereotax bite bar while cannula arm is positioned above the bitebar to prevent the need for repositioning during the procedure. Turn on light, position eyepieces, and focus scope on highest magnification (usually $4 \times$ ).

8. Prepare implant (Fig. 1): Modify the length of the fiber (which is in excess at the nonferrule [cranial] end; see Fig. 1A) to the specific depth necessary for delivering light into the structure of interest by following the steps below (see also Yizhar et al. 2011).

i. Using a ruled surface (quilting board) or small ruler as a guide, nick the fiber perpendicular to its long axis using the diamond knife (Fig. 1B).

At the target ventral depth, fibers cut to an appropriate length will have 0-2 $\mathrm{mm}$ of fiber separating the skull from the ferrule. This length is important, as fibers that are too long require large volumes of cement to be properly affixed to the skull, while those that are too short may not have enough travel length to achieve the desired coordinate before the ferrule comes into contact with the skull. 
L.E. Fenno et al.
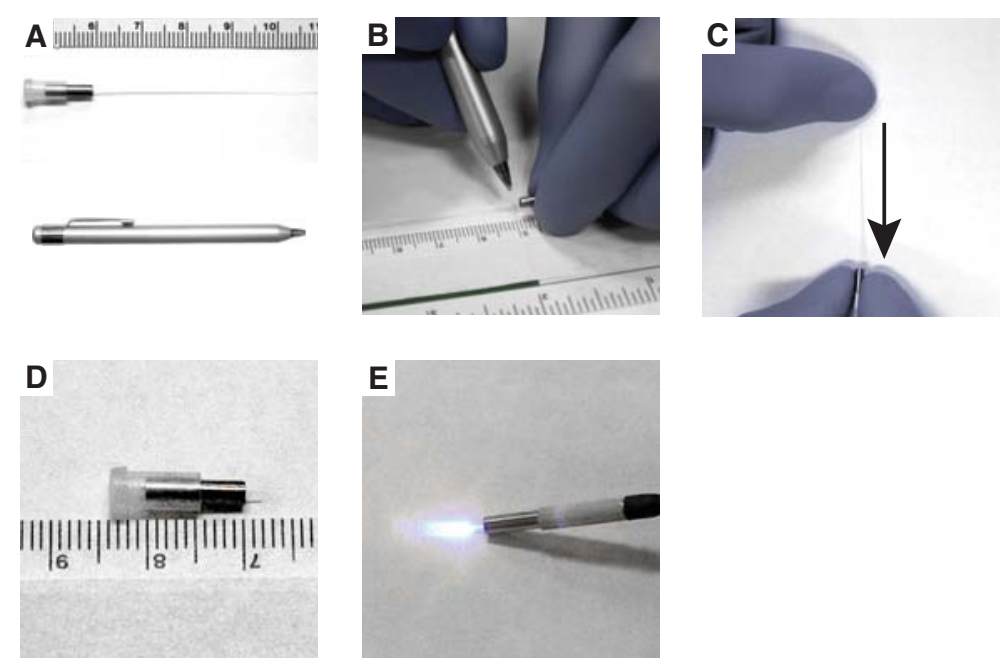

FIGURE 1. Preparing a fiber-optic implant. (A) Materials needed for this procedure include a ruler, stock fiber-optic implant, and diamond or steel carbide (pictured) knife. $(B, C)$ Trimming the fiber. See Step 8 for details. $(D)$ This process creates an implant of the desired length (here $\sim 2 \mathrm{~mm}$ ) capable of producing an even light spot $(E)$.

ii. While grasping the ferrule with your dominant hand, affix the excess length of fiber to a solid surface, such as a laboratory bench, using your nondominant thumb or a piece of laboratory tape.

iii. Pull straight back along the long axis of the implant (Fig. 1C) to initiate a clean break at the nick (Fig. 1D,E).

Flicking the fiber will also break it, but may create a jagged end that will cause uneven distribution of light.

Preparing the Mouse

9. Before beginning surgery, weigh the mouse to calculate the correct dosing of analgesics. Put the mouse into the anesthetic induction chamber and begin oxygen and isoflurane flow at $5 \%$.

The mouse should become immobile with deep breathing within 1 min.

10. Switch vaporizer outflow valve from induction chamber to nose cone and decrease isoflurane flow to $2 \%$. Remove mouse from induction box and place nose into nosecone. If upper teeth do not fall into bite bar hole, remove nose and try again. Repeat until teeth fall into place (properly positioned teeth will hold the jaw in the bite bar when you gently pull backward on the animal). Position mouse on heating pad, slide nosecone over snout, and secure nosecone with bottom screw.

11. Check tail and paw pinch reflexes.

See Troubleshooting.

12. Generously lubricate eyes.

13. Inject Buprenex (intraperitoneally) and Carprofen (subcutaneously).

14. Insert ear bars.

i. Loosen the right hold screw until the right ear bar slides freely. Gently push the right ear bar toward the animal while maneuvering the head so that it enters the area slightly in front of the ear canal. Tighten the right ear bar screw.

ii. Repeat with the left side.

iii. Once both right and left ear bars are gently sitting against the skull, loosen them slightly, one 
Downloaded from http://cshprotocols.cshlp.org/ on April 26, 2023 - Published by Cold Spring Harbor Laboratory Press

Optical Fiber Implant Surgery for Optogenetics

at a time, and, with gentle but firm pressure, press the ear bar into the skull. Tap the skull with your gloved finger to see whether it is immobilized.

When the skull is in the proper location, it will be completely immobilized.

iv. If the skull still moves when tapped, loosen the ear bars again, move the bite bar in the $\mathrm{A} / \mathrm{P}$ and $\mathrm{D} / \mathrm{V}$ axes and re-tighten the ear bars (see Fig. 2A for a properly positioned animal).

This is a skill that becomes easier with practice. See Troubleshooting.

15. Remove hair along incision line using scissors.

This helps to maintain a sterile field and facilitates closing the incision after the procedure is complete. In addition to scissors, chemical hair removal agents (e.g., Nair) or a small razor may be used.

16. Check for reflexes again, then create an incision.

The orientation, location, and length of the incision will depend on your exact procedure, but a midline incision along the A/P axis is usually sufficient. Take care to avoid the eyes in the anterior and neck and skull musculature in the posterior. Always cut skin directly on top of the skull. If you want to make a long posterior
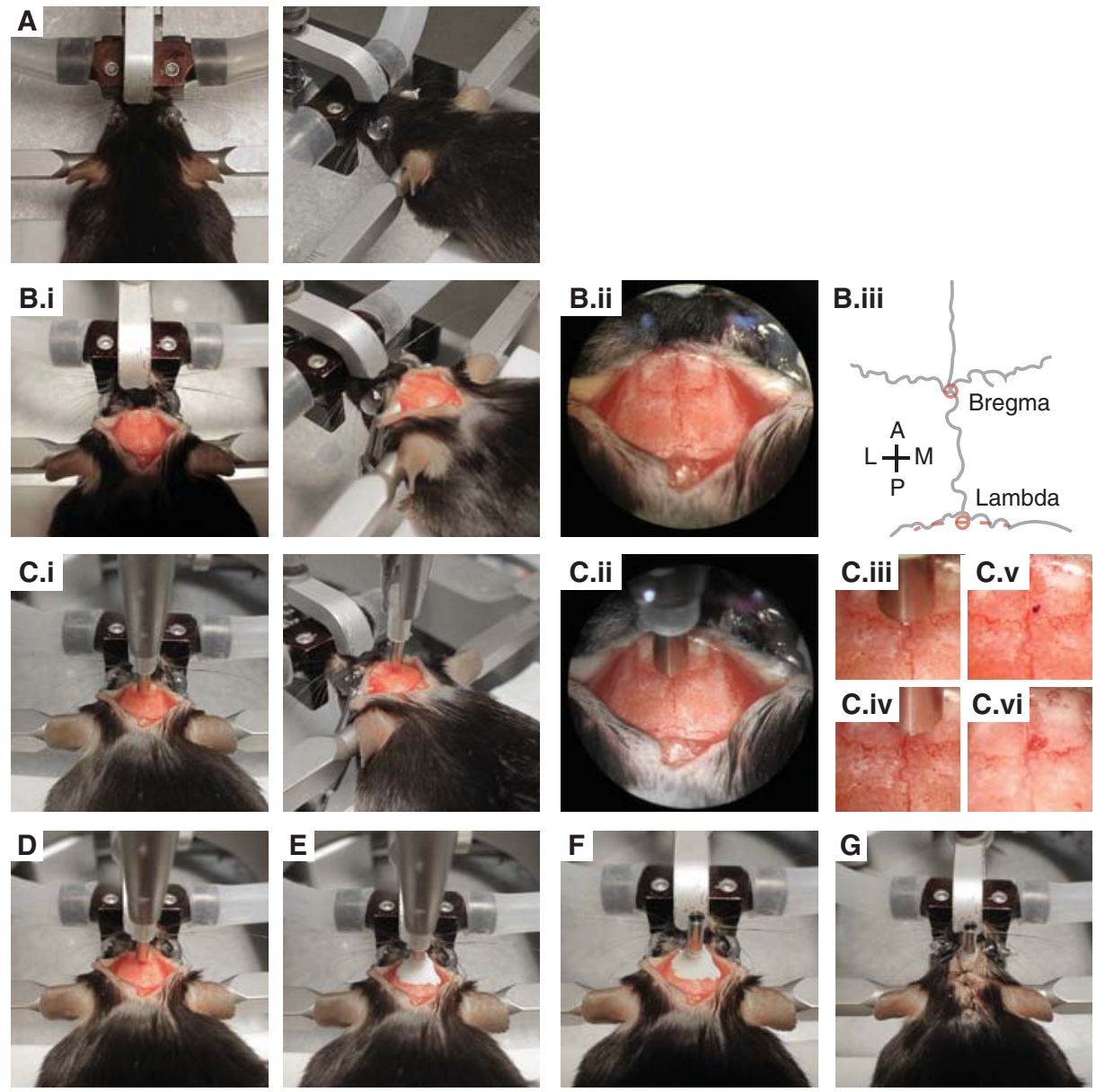

FIGURE 2. Surgical implantation of a fiber-optic implant. (A) The head of the animal has been immobilized in a stereotactic frame using bite bar and ear poles. Note eye lubrication. Here, the animal was prepared for surgery using a vaporized anesthetic, which is applied via the tubing and mask covering the snout. $(B)$ The scalp has been retracted from the skull surface to expose cranial sutures (B.i,ii). Alignment of bregma and lambda (B.iii drawn from $B$.ii) in the anterior-posterior (A/P) and dorsal-ventral (D/N) axes by manipulating the position of the skull within the stereotax. (C) The implant itself is used as a measuring device for the bregma-lambda alignment (C.i,ii). The final A/P and mediallateral $(\mathrm{M} / \mathrm{L})$ coordinates of the target region are calculated (C.iii) and the implant is moved to this location (C.iv). This location has been marked on the skull surface (C.v) and a small craniotomy performed (C.vi) to provide an access point for the implant to enter the brain. $(D)$ The implant after being inserted into the brain. $(E)$ The implant affixed to the skull with cement. $(F)$ After removal of the cannula. $(G)$ The sutured incision. 
L.E. Fenno et al.

incision, pinch the skin posterior to the skull, pull it anteriorly with forceps and continue to cut on top of the skull to avoid damaging neck musculature. Watch for any sign of sensation in the mouse during the incision and titrate the anesthetic level accordingly. See Troubleshooting.

17. Retract the scalp with hemostats to expose bregma and lambda skull landmarks (Fig. 2B.i-iii).

18. Use a scalpel to scrape the thin tissue layer from the top of the skull with short motions moving from medial to lateral.

This is a critical step that helps to prevent the loss of implants by improving adherence of cement to the skull.

19. Wet a cotton swab with saline and, again from center outward, clean the skull surface to remove bits of bone, tissue, and hair. Repeat as necessary and dry with a clean cotton swab when complete.

Performing the Craniotomy and Introducing the Fiber Implant

20. Affix the prepared implant to the stereotax cannula arm (Fig. 2C.i).

Depending on the arm, the implant may be too small to snuggly fit in the holder. If this is the case, an adapter may be fabricated. We either use laboratory tape or modify the plastic cap that protects the polished end of the implant.

21. Looking through the microscope, move the stereotaxic arm holding the implant until the tip of the fiber gently touches bregma (Fig. 2C.ii). Note these coordinates on the stereotax (or, in the case of digital stereotaxes, zero the readout).

Properly aligning skull landmarks to the stereotax is critical for the use of the coordinate system in neuroanatomy atlases and for consistency across procedures.

See Troubleshooting.

22. Move the implant to lambda taking care not to drag the implant across the skull. Note the new coordinates and subtract these numbers from the initial values to find the net distance traveled in the $\mathrm{A} / \mathrm{P}, \mathrm{M} / \mathrm{L}$, and $\mathrm{D} / \mathrm{V}$ planes.

Tolerable differences in the $M / L$ and $D / V$ planes will vary by experimental design; our general tolerance is $\pm 50 \mu \mathrm{m}$. The A/P distance between bregma and lambda will vary by animal, but is generally $4-4.5 \mathrm{~mm}$.

23. If the values are outside the acceptable tolerance bounds, make small movements one plane at a time.

i. Manipulate the D/V alignment via a screw anterior to the nose of the animal.

ii. Manipulate the M/L alignment by moving the ear bars.

To prevent loss of traction of the skull in the frame, loosen only one at a time and move it only slightly before tightening and matching the movement with the opposite ear bar.

24. After manipulating the position of the skull, move the fiber back to bregma and repeat the measurement process to assess the relative alignment of bregma and lambda until the skull position is acceptable.

25. After completing the alignment, move the implant to bregma and record the coordinates (Fig. 2C. iii). Use these bregma coordinates and the atlas coordinates of the region of interest to calculate relative coordinates of the region of interest.

26. Move the implant so that it is touching the skull at the $\mathrm{M} / \mathrm{L}$ and $\mathrm{A} / \mathrm{P}$ coordinates dorsal to the region of interest (Fig. 2C.iv).

27. Using local surface landmarks, a permanent marker, a needle, or some other mechanism, mark the area of skull that must be removed. Move the cannula arm out of the way to prevent damage to the fiber during drilling of the craniotomy (Fig. 2C.v).

28. Check tail and paw pinch reflexes.

See Troubleshooting. 
29. While looking through the microscope eyepieces, use the dental drill to begin removing bone above the region of interest. Begin by holding the drill so that the tip of the drill bit is visible through the microscope. Turn on the drill using the foot pedal, and once it is on, gently lower it to the surface of the skull.

The mouse skull is not thick. The outer layer of the skull is the thickest; the most dorsal layer is the softest. So, drill particularly slowly once you are approaching the surface of the brain. Be gentle and patient. Pressure is not necessary to create a craniotomy, but it will increase the likelihood that the drill will continue into the cortex and beyond if it suddenly breaks through the skull. If you find that you need to apply pressure to make progress, it is likely that you need to replace the drill bit. With mice, bits may need to be replaced after three or four craniotomies.

30. Stop periodically while creating the craniotomy to check that it will be in the correct location for the implant and to ensure that the animal shows no signs of sensation. Adjust drilling as necessary.

31. If the animal begins to bleed, use your free hand and an absorption spear to prevent the blood from obscuring your view of the craniotomy.

Some locations are more prone to bleeding than others. This especially includes those near the midline, which may tear larger blood vessels.

See Troubleshooting.

32. Stop drilling once you are able to clearly view the surface of the brain, including vessels (Fig. 2C.vi).

It is possible that skull fragments and/or dura will remain above the cortex. These will impact the entrance of the implant and should be removed. Various tools suitable for this task, such as fine forceps, are available from surgical suppliers, but we find that a small gauge needle bent to a 90-degree angle also works well.

33. Create additional craniotomies as needed. After all drilling is complete, remove bone dust, blood, and hair from the surface of the skull using a cotton swab wetted with saline. Dry with a new cotton swab.

34. Scrape the surface of the skull with a scalpel and repeat rinse and dry (Step 33) to create a smooth, clean surface.

This is critical to prevent implant loss.

35. After the skull is cleaned, visually inspect the craniotomy to ensure that it is free of debris and clotted blood. If it is not, clean it using the needle or an absorption spear.

36. While observing through the microscope, move the tip of the implant to the surface of the cortex. It is critical to watch the implant enter the brain to ensure that skull fragments do not deflect it. This is a common cause of mistargeting.

37. In slow movements, ease the implant into the brain, watching to ensure that it is penetrating the brain parenchyma and not simply compressing it (Fig. 2D). Check the depth on the D/V arm as you lower the implant to prevent damage to the region of interest caused by overshooting the ventral target coordinate.

Securing the Implant

38. Check tail and paw pinch reflexes. Re-lubricate eyes as needed.

See Troubleshooting.

39. If necessary, soak up any remaining blood using an absorption spear.

The skull should still be clean and dry from the previous cleaning. Perform this step with care, because properly securing the implant requires direct contact between the skull and cement.

40. Apply the first layer of dental cement.

i. Line the ceramic mixing stand with foil.

The foil makes for easy cleanup. 
L.E. Fenno et al.

ii. Using two scoops of metabond powder, follow the manufacturer's instructions to prepare the cement.

This cement sets rapidly, so quick application after mixing is critical.

iii. After the cement has begun to thicken, use the supplied brush to cover a small $(\sim 4 \times 4 \mathrm{~mm})$ section of the skull surrounding the implant with cement.

iv. Add cement directly to the sides of the implant, taking care not to cement the implant to the cannula holder arm of the stereotax and not to get cement in the rodent's eyes.

Keep in mind that the ferrule is connected to the patch fiber by a sleeve and thus at least 3-5 mm of the top of the ferrule will need to remain cement free.

v. Add more cement to connect the previously applied cement on the ferrule and skull, making sure all sides are evenly coated to produce a tapered cone shape with its widest surface on the skull.

41. Wait for the cement to dry (Fig. 2E). Test for dryness by gently probing the cement with forceps. Dried cement will not dent or give under pressure.

See Troubleshooting.

42. Once this layer has dried, remove the cannula holder (Fig. $2 \mathrm{~F}$ ).

It may be useful to use the handle of a small pair of forceps to pry apart the two halves of the cannula holder while raising it free of the implant.

See Troubleshooting.

43. (Optional) Add a second layer of adhesive on top of the first to create a smooth surface where the cement meets the scalp and decrease irritation.

Ortholet is inexpensive and works well for this purpose. It takes significantly longer to dry than Metabond, but allows more time for shaping. To incorporate this step into your procedure, repeat the steps used for the Metabond application, ensuring that you smooth and contour the surface and cover any sharp edges.

Closing the Incision and Recovering the Animal

44. Check tail and paw reflexes and eye lubrication. See Troubleshooting.

45. Use suture to close the scalp both anterior and posterior of the implant (Fig. 2G). Leave some slack between the last stitch and the mound of cement to avoid tension on the suture.

Some protocols allow for the use of skin glue to close incisions.

46. Loosen the ear bars and retract them from the animal. Unscrew and push the nose cone away from the mouse. Carefully lift the upper snout from the bite bar, taking care to lift and not pull to avoid damaging the teeth.

47. Place the animal in a heated recovery chamber. Ensure that it will not allow the animal to escape once it has recovered.

48. Turn off isoflurane and oxygen and clean the surgical area and tools. Place sharps, including scalpel blades, suture needles, and syringes in a sharps container for disposal.

49. Once the animal has fully recovered, return it to its cage.

We provide postoperative animals with edible antibiotic and analgesic pellets. Depending on your protocol, animals may need to be housed separately after surgery until healing is complete. This may also prevent "groomers" in the cage from re-opening and infecting the incision.

Preparing for Stimulation

When operating lasers, always use proper precautions as stated by the manufacturer and your institutional occupational safety office.

50. Attach a patch fiber to the laser via FC/PC connectors. 
Stimulating the Animal

These use a male/female coupling system and it may be necessary to use an adaptor section to couple some lasers/components.

51. Ensure that the patch fiber has a zirconia sleeve attached to the ferrule.

This sleeve holds the patch fiber and animal implant flush with each other to allow maximal transmission of laser light into the brain.

52. Secure the fiber so that any emitted light will be directed in a safe direction.

53. Couple the analog output of a standard function generator to the analog input of the laser base.

54. Switch on the function generator and program a stimulation paradigm consisting of the frequency (lasers pulses per second), pulse width (length of each laser pulse), $V_{\max }$ (maximum output voltage, usually $5 \mathrm{~V}$ ), and $V_{\min }$ (minimum output voltage; see Fig. 3 for description of variables).

55. Switch on the laser base power, switch the key to on, turn on the function generator output, and modulate the laser base dial to reach the desired irradiance (as measured by a power meter).

56. Switch the function generator output off.

For some lasers, this may cause the laser to default to "on." If that occurs, the laser safety key may be used to turn off the laser power.

57. Immobilize the animal by grasping its tail with one hand (Fig. $4 \mathrm{~A}$ ) and using the thumb and index finger of your other hand to firmly push the animal to the work surface while moving your fingers from the mid-back starting position (Fig. 4B) toward the head. Remember, you are bigger than the mouse.

58. When you reach the shoulder blades, slightly loosen your thumb and index finger, move them beyond the shoulders, and pinch and draw back the skin from underneath the ears/jaw toward the back of the neck to immobilize the head of the animal (Fig. 4C).

Doing this for prolonged periods or with excessive force may choke the animal. Only use enough force to immobilize the head.

59. Once the head is immobilized, release the tail and pick up the zirconia sleeve at the ferrule end of the patch cable (Fig. 4D). Slide this over the ferrule of the implant (Fig. 4E). Look at the slit in the zirconia sleeve to ensure that the two ferrules are in contact.

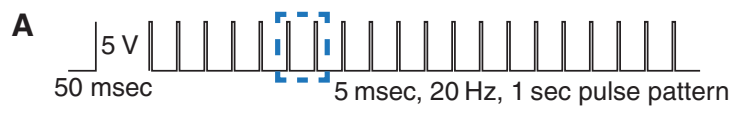

B

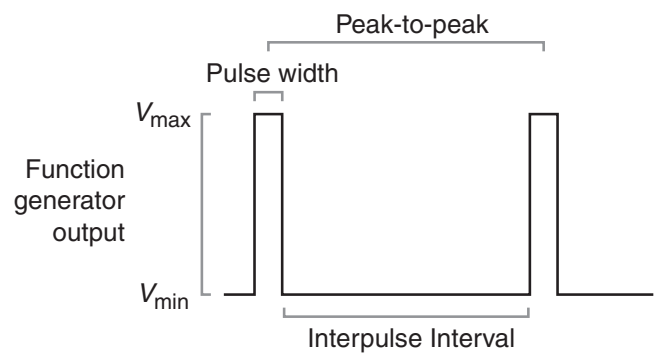

FIGURE 3. Components of the stimulation paradigm. $(A)$ The output of a function generator programmed to produce a typical $20-\mathrm{Hz}, 5-\mathrm{msec}$ pulse width, $5-\mathrm{V}$ square pulse pattern is diagrammed. $(B)$ The common Laser output variable names of these parameters are indicated in the focus on two pulses from the example pulse pattern (top). Resulting laser output (middle) should only $\frac{1}{\text { Interpulse interval + Pulse width }}=\frac{1}{\text { Peak-to-Peak }}=\lambda_{\text {stim }}$ occur during square pulses of the stimulation train at a predetermined frequency (bottom). 
L.E. Fenno et al.
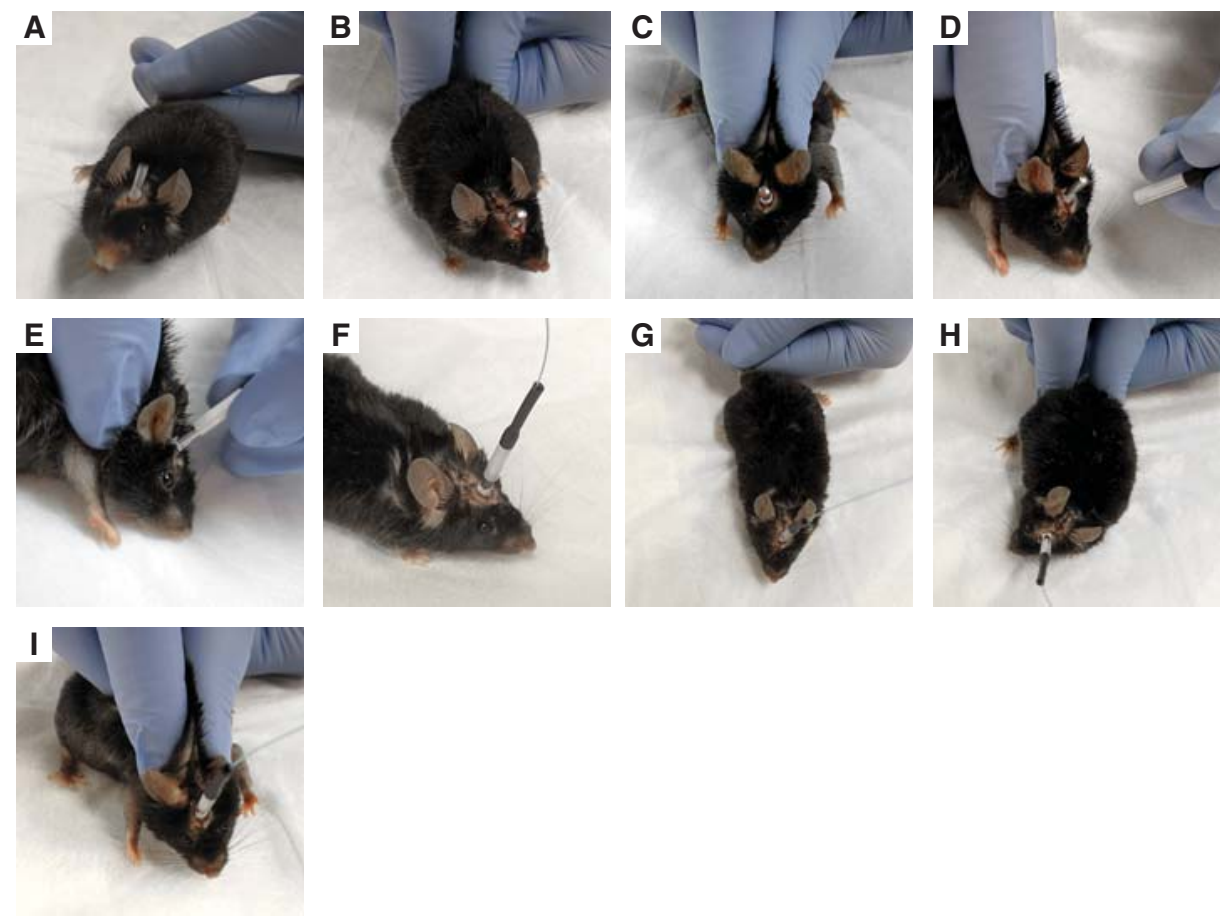

FIGURE 4. Connecting a patch fiber to the implant. $(A)$ The mouse is initially grasped at the base of the tail with one hand. $(B)$ The rump of the animal is pressed with the index finger and thumb of the other hand. (C) The index finger and thumb slide to and move beyond the shoulders, and then the head is immobilized by stretching the skin between the shoulders and the base of the skull. $(D)$ Preparing to insert the patch cord. $(E)$ The patch cable is connected to the implant. $(F)$ Animal is released and observed. $(G-I)$ To remove the patch cable, the handling procedure is repeated.

60. Release the animal into a holding cage or behavioral arena (Fig. 4F).

61. Turn on the laser and function generator, and observe! See Troubleshooting.

62. After behavior is complete, repeat the immobilization process and disconnect the patch fiber by grasping the patch fiber ferrule/zirconia sleeve and twisting to remove (Fig. 4G-I).

Improper immobilization will result in twisting of the animal's neck.

Analyzing the Data

63. Analyze behavioral data using standard methods.

64. Isolate the brain of the animal using standard and humane techniques.

We use trans-cardial perfusion in animals anesthetized with pentobarbital. The implant can be removed after perfusion by fixing the skull in place using one finger on the snout and one on the neck while using a large hemostat with the other hand to pull the implant out by the ferrule.

65. Section the brain to locate the implant track.

Standard implants are $200 \mu \mathrm{m}$ and may be located across multiple serial sections at 40-45- $\mu \mathrm{m}$ cuts.

66. Analyze gene expression at the stimulation site.

Thy 1:18 animals express a fusion ChR2-YFP gene. Expression may be analyzed using an epifluroescence microscope.

67. If more surgeries will be performed, compare your actual implantation site to your target and adjust coordinates as necessary. Mouse neuroanatomy and the relationship between specific brain structures and skull sutures varies by strain and age, and anatomy atlases are therefore an accurate, but not precise, starting point for designing surgical procedures. 
Problem (Steps 11, 16, 28, 38, and 44): The mouse has a toe pinch during surgery.

Solution: Temporarily increase isoflurane concentration as needed to stabilize the animal's condition and eliminate toe pinch reflex. Bring back down to $2 \%$ for maintenance for the duration of the surgery.

Problem (Step 14): Skull is unstable in the ear bars.

Solution: Try moving the bite bar in the $\mathrm{A} / \mathrm{P}$ and $\mathrm{D} / \mathrm{V}$ directions using the knobs on top of the mouth piece and at the very back of the stereotax-there will be a sweet spot where the skull falls into place securely.

Problem (Step 21): You have trouble locating bregma or lambda landmarks on the skull.

Solution: Wait until the skull is thoroughly dry (you can wipe the skull with dilute hydrogen peroxide to help visualize skull plate intersections). Use forceps to lightly tap skull plates to help see where they intersect. As a guideline, bregma and lambda are roughly $4.5 \mathrm{~mm}$ apart.

Problem (Step 31): The craniotomy will not stop bleeding.

Solution: Soak up excess blood with a cotton swab. You can also try wetting a small piece of cotton with saline and place it in the craniotomy to stem the blood flow and allow it to clot. If the mouse has lost a lot of blood, give it $1 \mathrm{~mL}$ of saline or lactated Ringer's injected intraperitoneally. To prevent light blockage, make sure to clear all fresh and dried blood before lowering the optical fiber.

Problem (Step 41): Metabond cement is not drying properly.

Solution: It is possible that you did not add enough catalyst or are using one that is too old. Re-make the cement with an extra drop of catalyst making sure everything is thoroughly mixed and apply over existing layer.

Problem (Step 42): Cannula cemented to cannula holder.

Solution: The best solution to this problem is to avoid it in the first place. However, in the event that your cannula becomes one with the cannula holder, potential remedies include using the drill to pulverize cement that is joining the two, using small amounts of solvent to dissolve the cement, and using the handle portion of a flat surgical tool (e.g., scalpel, forceps) to pry the jaws of the cannula holder apart. Good luck!

Problem (Step 42): Cannula holder would not lift up from implant, although it is not cemented.

Solution: Wedge forceps between the two halves of the cannula holder to pry them open while simultaneously lifting cannula holder.

Problem (Step 61): Mouse has a seizure when the laser is turned on.

Solution: Try lowering the light power or frequency and/or pulse width of stimulation.

Problem (Step 61): Nothing happens with stimulation/ mouse does not move.

Solution: Try higher light power, frequency, or pulse width. You can also try putting the mouse in different environments (i.e., a safe familiar home cage environment) to reduce anxiety and increase locomotion.

\section{REFERENCES}

Adesnik H, Scanziani M. 2010. Lateral competition for cortical space by layer-specific horizontal circuits. Nature 464: 1155-1160.

Arenkiel BR, Peca J, Davison IG, Feliciano C, Deisseroth K, Augustine GJ, Ehlers MD, Feng G. 2007. In vivo light-induced activation of neural circuitry in transgenic mice expressing channelrhodopsin-2. Neuron 54: 205-218.

Fenno L, Yizhar O, Deisseroth K. 2011. The development and application of optogenetics. Annu Rev Neurosci 34: 389-412. 
L.E. Fenno et al.

Gradinaru V, Thompson KR, Zhang F, Mogri M, Kay K, Schneider MB, Deisseroth K. 2007. Targeting and readout strategies for fast optical neural control in vitro and in vivo. J Neurosci 27: 14231-14238.

Greenshaw AJ. 1997. A simple technique for determining stereotaxic coordinates for brain implantation of probes at rotated angles in one or two planes. J Neurosci Methods 78: 169-172.

Madisen L, Zwingman TA, Sunkin SM, Oh SW, Zariwala HA, Gu H, Ng LL, Palmiter RD, Hawrylycz MJ, Jones AR, et al. 2010. A robust and highthroughput Cre reporting and characterization system for the whole mouse brain. Nat Neurosci 13: 133-140.
Madisen L, Mao T, Koch H, Zhuo JM, Berenyi A, Fujisawa S, Hsu YW, Garcia AJ 3rd, Gu X, Zanella S, et al. 2012. A toolbox of Cre-dependent optogenetic transgenic mice for light-induced activation and silencing. Nat Neurosci 15: 793-802.

Witten IB, Steinberg EE, Lee SY, Davidson TJ, Zalocusky KA, Brodsky M, Yizhar O, Cho SL, Gong S, Ramakrishnan C, et al. 2011. Recombinase-driver rat lines: Tools, techniques, and optogenetic application to dopamine-mediated reinforcement. Neuron 72: 721-733.

Yizhar O, Fenno LE, Davidson TJ, Mogri M, Deisseroth K. 2011. Optogenetics in neural systems. Neuron 71: 9-34. 


\section{Mapping Anatomy to Behavior in Thy1:18 ChR2-YFP Transgenic Mice Using Optogenetics}

Lief E. Fenno, Lisa A. Gunaydin and Karl Deisseroth

Cold Spring Harb Protoc; doi: 10.1101/pdb.prot075598

\begin{tabular}{cc}
$\begin{array}{r}\text { Email Alerting } \\
\text { Service }\end{array}$ & Receive free email alerts when new articles cite this article - click here. \\
\hline $\begin{array}{c}\text { Subject } \\
\text { Categories }\end{array}$ & $\begin{array}{c}\text { Browse articles on similar topics from Cold Spring Harbor Protocols. } \\
\text { Behavioral Assays (99 articles) } \\
\text { Mouse (437 articles) } \\
\text { Neuroscience, general (357 articles) }\end{array}$ \\
\hline
\end{tabular}

